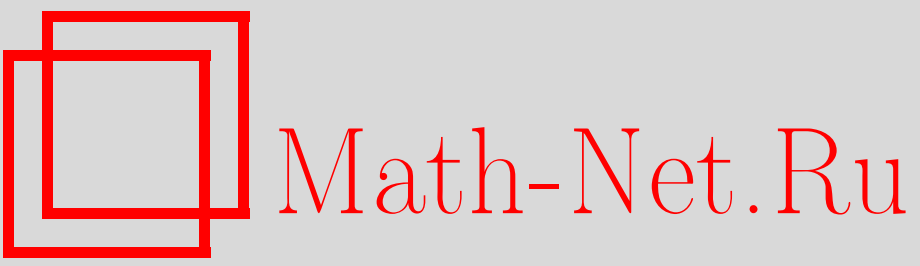

С. М. Ташпулатов, Одномагнонные системы в изотропной примесной негейзенберговской ферромагнитной модели, ТМФ, 2005, том 142, номер 1, 83-92

DOI: https://doi.org/10.4213/tmf1768

Использование Общероссийского математического портала Math-Net.Ru подразумевает, что вы прочитали и согласны с пользовательским соглашением

http://www.mathnet.ru/rus/agreement

Параметры загрузки:

IP: 3.85 .7 .115

26 апреля 2023 г., 12:27:31 
ТЕОРЕТИЧЕСКАЯ

И МАТЕМАТИЧЕСКАЯ

ФИЗИКА

Том 142, № 1

январь, 2005

(C) 2005 г.

С. М. Ташпулатов*

\title{
ОДНОМАГНОННЫЕ СИСТЕМЫ В ИЗОТРОПНОЙ ПРИМЕСНОЙ НЕГЕЙЗЕНБЕРГОВСКОЙ ФЕРРОМАГНИТНОЙ МОДЕЛИ
}

\begin{abstract}
Рассматривается одномагнонная система в изотропной примесной негейзенберговской модели с произвольным значением спина. Исследуются спектр и локализованные примесные состояния этой системы на $\nu$-мерной целочисленной решетке $Z^{\nu}$. Показано, что система имеет не более трех типов локализованных примесных состояний (без учета кратности вырождений их энергии). Найдены области сушествования этих состояний и вычислены кратности вырождений их энергии.
\end{abstract}

Ключевые слова: примесные состояния, решетка, взаимодействия, операторы рождения и уничтожения.

Применение пленок в различных областях физики и техники обусловливает значительный интерес к изучению локализованных примесных состояний (ЛПС) магнетиков.

ЛПС в ферромагнетике Гейзенберга с ферромагнитной и антиферромагнитной примесями изучались во многих работах (см., например, [1]), в которых подробно рассматривались случаи линейной или кубической решетки. Было показано, что в линейном случае сушествуют два типа ЛПС, а в случае кубической решетки - три типа ЛПС.

В работе [2] рассматривался случай $\nu$-мерной решетки и было доказано, что в $\nu$-мерном случае система имеет не более трех типов ЛПС (без учета кратности вырождений их энергии). Показано, что с изменением значений параметров гамильтониана количество ЛПС системы изменяется. Найдены их области сушествования. Оказывается, что в этом случае ЛПС системы этих трех типов соответственно невырожденны, $\nu$ и $(\nu-1)$-кратно вырожденны.

В теоретических исследованиях магнитоупорядоченных систем и при интерпретации экспериментальных фактов, полученных для этих систем, обычно исходили из гейзенберговского обменного гамильтониана (для произвольного значения спина $s$ )

$$
H_{1}=J \sum_{m, \tau}\left(\mathbf{S}_{m} \mathbf{S}_{m+\tau}\right)
$$

\footnotetext{
* Институт ядерной физики АН РУз, Ташкент, Узбекистан. E-mail: toshpul@inp.uz
} 
где $J$ - параметр билинейного обменного взаимодействия между атомами ближайших соседей, $\mathbf{S}_{m}$ - оператор атомного спина величины $s$ в $m$-м узле $\nu$-мерной решетки $Z^{\nu}$, а суммирование по индексу $\tau$ обозначает суммирование по ближайшим соседям.

В действительности для произвольного значения спина $s$ изотропный спиновый обменный гамильтониан имеет вид [3]

$$
H=\sum_{m, \tau} \sum_{n=1}^{2 s} J_{n}\left(\mathbf{S}_{m} \mathbf{S}_{m+\tau}\right)^{n}
$$

где $J_{n}$ - параметры мультипольных обменных взаимодействий между атомами ближайших соседей. Гамильтониан (2) совпадает с гамильтонианом (1) только при $s=1 / 2$, а при $s>1 / 2$ появляются члены, содержашие более высокие степени $\left(\mathbf{S}_{m} \mathbf{S}_{m+\tau}\right)$, которые необходимо учитывать при исследовании магнетиков со значением спина $s>1 / 2$. Гамильтониан (2) называется негейзенберговским.

В течении многих десятилетий модель Гейзенберга была основой, на которой развивалась теория магнетизма. Следует отметить, что число магнетиков, близких к идеальным гейзенберговским, не так уж велико. Для магнетиков со значением спина $s>1 / 2$ модель Гейзенберга недостаточно хорошо описывает их свойства. Магнитные свойства негейзенберговских магнетиков сильно отличаются от свойств гейзенберговских магнетиков, а расчет обмена высших порядков по спину несравнимо сложнее гейзенберговскоГо.

В данной работе рассматриваются одномагнонные состояния в изотропной примесной негейзенберговской ферромагнитной модели с произвольным значением спина $s$. Найдены изменение количества ЛПС, их энергии, кратности вырождений этих энергий, области их существования с изменением значений параметров гамильтониана. Насколько нам известно, спектр и ЛПС одномагнонных систем в негейзенберговской изотропной примесной ферромагнитной модели с произвольным значением спина $s$ с учетом мультипольных обменных взаимодействий в литературе не обсуждались.

Гамильтониан рассматриваемой системы имеет вид

$$
H=-\sum_{m, \tau} \sum_{n=1}^{2 s} J_{n}\left(\mathbf{S}_{m} \mathbf{S}_{m+\tau}\right)^{n}-\sum_{\tau} \sum_{n=1}^{2 s}\left(J_{n}^{0}-J_{n}\right)\left(\mathbf{S}_{0} \mathbf{S}_{\tau}\right)^{n}
$$

Здесь $J_{n}>0$ - параметры мультипольного обменного взаимодействия между ближайшими атомами решетки, $J_{n}^{0}$ - параметры мультипольного обменного взаимодействия между атомами и примесями, $\tau= \pm e_{j}$, где $e_{j}$ - единичные орты, $j=1,2, \ldots, \nu$, т.е. суммирование по $\tau$ означает суммирование по ближайшим соседям, $\mathbf{S}_{m}=\left(S_{m}^{x} ; S_{m}^{y} ; S_{m}^{z}\right)-$ оператор атомного спина величины $s$ в узле $m$. Положим $S_{m}^{ \pm}=S_{m}^{x} \pm i S_{m}^{y}$, где $S_{m}^{-}$и $S_{m}^{+}-$соответственно операторы рождения и уничтожения магнона в узле $m$.

Гамильтониан (3) действует в симметрическом пространстве Фока $\mathcal{H}$. Обозначим через $\varphi_{0}$ вектор, называемый вакуумным и однозначно определяемый условиями 
$S_{m}^{+} \varphi_{0}=0, S_{m}^{z} \varphi_{0}=s \varphi_{0},\left\|\varphi_{0}\right\|=1$. Вектор $S_{m}^{-} \varphi_{0}$ описывает состояние системы одного магнона, находящегося в узле $m$, со значением спина $s$. Векторы $\left\{S_{m}^{-} \varphi_{0}\right\}$ образуют ортонормальную систему. Замыкание пространства, натянутого на эти векторы, обозначим через $\mathcal{H}_{1}$, оно евклидово относительно естественного скалярного произведения и называется пространством одномагнонных состояний. Имеет место

ПРЕДЛОЖЕНИЕ 1. Пространство $\mathcal{H}_{1}$ инвариантно относительно оператора $H$. Оператор $H_{1}=H / \mathcal{H}_{1}$ является ограниченным самосопряженным оператором, который порождает ограниченный самосопряженный оператор $\bar{H}_{1}$, действующий в пространстве $l_{2}\left(Z^{\nu}\right)$ по формуле

$$
\begin{aligned}
\left(\bar{H}_{1} f\right)(p)= & \sum_{k=1}^{2 s}(-1)^{k+1} J_{k} s^{k} \sum_{\tau} 2^{k-1}[2 f(p)-f(p+\tau)-f(p-\tau)]+ \\
& +\sum_{k=1}^{2 s}(-1)^{k+1}\left(J_{k}^{0}-J_{k}\right)(2 s)^{k} \sum_{\tau}(f(\tau)-f(0))\left(\delta_{p, \tau}-\delta_{p, 0}\right), \quad p \in Z^{\nu},
\end{aligned}
$$

где $\delta_{k, j}$ - символ Кронекера, а суммирование по $\tau$ означает суммирование по ближайшим соседям. Сам оператор $H_{1}$ действует на вектор

$$
\psi=\frac{1}{\sqrt{2 s}} \sum_{p} f(p) S_{p}^{-} \varphi_{0}, \quad \psi \in \mathcal{H}_{1}
$$

по формуле

$$
H_{1} \psi=\sum_{p}\left(\bar{H}_{1} f\right)(p) \frac{1}{\sqrt{2 s}} S_{p}^{-} \varphi_{0}
$$

Предложение 1 доказывается с использованием известных коммутационных соотношений между операторами $S_{m}^{+}, S_{p}^{-}$и $S_{q}^{z}$.

Лемма 1. Спектры операторов $H_{1}$ и $\bar{H}_{1}$ совпадают.

ДокАЗАТЕльСтво. Так как операторы $H_{1}$ и $\bar{H}_{1}$ являются ограниченными самосопряженными операторами, то, если $\lambda \in \sigma\left(H_{1}\right)$, из критерия Вейля [4] следует сушествование последовательности $\left\{\psi_{n}\right\}_{n=1}^{\infty}$ такой, что $\left\|\psi_{n}\right\|=1$ и $\lim _{n \rightarrow \infty}\left\|\left(H_{1}-\lambda\right) \psi_{n}\right\|=0$. Положим $\psi_{n}=\frac{1}{\sqrt{2 s}} \sum_{p} f_{n}(p) S_{p}^{-} \varphi_{0}$. Тогда

$$
\begin{aligned}
\left\|\left(H_{1}-\lambda\right) \psi_{n}\right\|^{2} & =\left(\left(H_{1}-\lambda\right) \psi_{n},\left(H_{1}-\lambda\right) \psi_{n}\right)= \\
& =\sum_{p}\left\|\left(\bar{H}_{1}-\lambda\right) f_{n}(p)\right\|^{2}\left(\frac{1}{\sqrt{2 s}} S_{p}^{-} \varphi_{0}, \frac{1}{\sqrt{2 s}} S_{p}^{-} \varphi_{0}\right)= \\
& =\left\|\left(\bar{H}_{1}-\lambda\right) F_{n}\right\|^{2} \cdot\left(\frac{1}{2 s} S_{p}^{+} S_{p}^{-} \varphi_{0}, \varphi_{0}\right)= \\
& =\left\|\left(\bar{H}_{1}-\lambda\right) F_{n}\right\|^{2}\left(\frac{1}{2 s} \cdot 2 s \varphi_{0}, \varphi_{0}\right)=\left\|\left(\bar{H}_{1}-\lambda\right) F_{n}\right\|^{2} \rightarrow 0
\end{aligned}
$$


при $n \rightarrow \infty$. Здесь $F_{n}=\sum_{p} f_{n}(p)$. Отсюда следует, что $\lambda \in \sigma\left(\bar{H}_{1}\right)$. Следовательно, $\sigma\left(H_{1}\right) \subset \sigma\left(\bar{H}_{1}\right)$. Обратно, пусть $\bar{\lambda} \in \sigma\left(\bar{H}_{1}\right)$, тогда в силу того же критерия Вейля сушествует последовательность $\left\{F_{n}\right\}_{n=1}^{\infty}$ такая, что $\left\|F_{n}\right\|=1$ и $\lim _{n \rightarrow \infty}\left\|\left(\bar{H}_{1}-\bar{\lambda}\right) F_{n}\right\|=0$. Полагая

$$
F_{n}=\sum_{p} f_{n}(p), \quad\left\|F_{n}\right\|=\left(\sum_{p}\left|f_{n}(p)\right|^{2}\right)^{1 / 2},
$$

имеем, что $\left\|\psi_{n}\right\|=\left\|F_{n}\right\|=1$ и $\left\|\left(\bar{H}_{1}-\bar{\lambda}\right) F_{n}\right\|=\left\|\left(H_{1}-\bar{\lambda}\right) \psi_{n}\right\| \rightarrow 0$ при $n \rightarrow \infty$. Это означает, что $\bar{\lambda} \in \sigma\left(H_{1}\right)$, т.е. $\sigma\left(\bar{H}_{1}\right) \subset \sigma\left(H_{1}\right)$. Эти два включения дают равенство $\sigma\left(H_{1}\right)=$ $\sigma\left(\bar{H}_{1}\right)$.

Видно, что если вектор (5) есть собственная функция оператора $H_{1}$ с собственным значением $z \notin G_{\nu}$, то вектор $F=\sum_{p} f(p)$ является собственной функцией для оператора $\bar{H}_{1}$ с тем же собственным значением $z \notin G_{\nu}$, и это собственное значение имеет одну и ту же кратность. Поэтому для исследования спектра оператора $H_{1}$ достаточно исследовать спектр оператора $\bar{H}_{1}$, действуюшего в $l_{2}\left(Z^{\nu}\right)$ по формуле (4).

Спектр и ЛПС оператора $H_{1}$ легко изучить в его квазиимпульсном представлении. Обозначим через $\mathcal{F}$ преобразование Фурье

$$
\mathcal{F}: l_{2}\left(Z^{\nu}\right) \rightarrow L_{2}\left(T^{\nu}\right)
$$

Здесь $T^{\nu}-\nu$-мерный тор, снабженньй нормированной мерой Лебега $d \lambda: \lambda\left(T^{\nu}\right)=1$.

Пусть

$$
\mathcal{F}: \mathcal{H}_{1} \rightarrow \widetilde{\mathcal{H}}_{1} \equiv L_{2}\left(T^{\nu}\right) .
$$

ПРЕДЛОЖЕниЕ 2. Преобразование Фурье переводит оператор $\bar{H}_{1}$ в оператор $\widetilde{H}_{1}=\mathcal{F} \bar{H}_{1} \mathcal{F}^{-1}$, который действует в пространстве $\widetilde{\mathcal{H}}_{1}$ по формуле

$$
\left(\widetilde{H}_{1} f\right)(x)=p(s) h(x) f(x)+q(s) \int_{T^{\nu}} h_{1}(x, t) f(t) d t,
$$

2วe

$$
h(x)=\nu-\sum_{i=1}^{\nu} \cos x_{i}, \quad h_{1}(x, t)=\nu+\sum_{i=1}^{\nu}\left[\cos \left(x_{i}-t_{i}\right)-\cos x_{i}-\cos t_{i}\right] .
$$

3дecb

$$
p(s)=-2 \sum_{k=1}^{2 s}(-2 s)^{k} J_{k}, \quad q(s)=-2 \sum_{k=1}^{2 s}(-2 s)^{k}\left(J_{k}^{0}-J_{k}\right) .
$$

Предложение 2 доказывается непосредственным применением преобразования Фурье к (4).

Ясно, что непрерьвный спектр оператора $\widetilde{H}_{1}$ не зависит от функции $q(s) h_{1}(x, t)$ и заполняет весь отрезок $G_{\nu}=\left[m_{\nu} ; M_{\nu}\right]$, где

$$
m_{\nu}=\min _{x \in T^{\nu}} p(s) h(x), \quad M_{\nu}=\max _{x \in T^{\nu}} p(s) h(x) .
$$


Собственная функция $\varphi \in L_{2}\left(T^{\nu}\right)$ оператора $\widetilde{H}_{1}$, отвечаюшая собственному значению $z \notin G_{\nu}$, называется ЛПС оператора $\widetilde{H}_{1}$, а величина $z$ - энергией этого состояния.

Рассмотрим оператор $K_{\nu}(z)$, действуюший в пространстве $\widetilde{\mathcal{H}}_{1}$ по формуле

$$
\left(K_{\nu}(z) f\right)(x)=\int_{T^{\nu}} \frac{h_{1}(x ; t)}{p(s) h(t)-z} f(t) d t, \quad x, t \in T^{\nu} .
$$

Он является вполне непрерывным оператором в пространстве $\widetilde{\mathcal{H}}_{1}$ для значений $z \notin G_{\nu}$.

Пусть

$$
\begin{aligned}
\Delta_{\nu}(z)= & \left(1+q(s) \int_{T^{\nu}} \frac{\left(1-\cos t_{1}\right)\left(\nu-\sum_{i=1}^{\nu} \cos t_{i}\right) d t}{p(s) h(t)-z}\right) \times \\
& \times\left(1+q(s) \int_{T^{\nu}} \frac{\sin ^{2} t_{1} d t}{p(s) h(t)-z}\right)^{\nu} \times \\
& \times\left(1+\frac{q(s)}{2} \int_{T^{\nu}} \frac{\left(\cos t_{1}-\cos t_{2}\right)^{2} d t}{p(s) h(t)-z}\right)^{\nu-1},
\end{aligned}
$$

где $d t=d t_{1} d t_{2} \ldots d t_{\nu}$.

Лемма 2. Число $z_{0} \notin G_{\nu}$ является собственным значением оператора $\widetilde{H}_{1}$ тогда и только тогда, когда оно является нулем функиии $\Delta_{\nu}(z)$, m.е. $\Delta_{\nu}\left(z_{0}\right)=0$.

ДокАЗАТЕльСтво. В нашем случае уравнение для собственных значений является интегральным уравнением с вырожденным ядром. Поэтому оно эквивалентно системе линейных однородных уравнений. Известно, что система линейных однородных алгебраических уравнений имеет нетривиальное решение тогда и только тогда, когда детерминант системы равен нулю, а детерминант системы есть функция $\Delta_{\nu}(z)$.

Обозначим через $\Omega$ множество значений всех пар $\omega=(p(s), q(s))$ и при $\nu=1$ введем следуюшие его подмножества:

$$
\begin{array}{ll}
A_{1}=\{\omega: p(s)>0,-p(s) \leqslant q(s)<0\}, & A_{2}=\{\omega: p(s)>0, q(s)>-p(s)\}, \\
A_{3}=\{\omega: p(s)<0, q(s)>p(s)\}, & A_{4}=\{\omega: p(s)>0, p(s)<q(s)\}, \\
A_{5}=\{\omega: p(s)>0,0<q(s) \leqslant p(s)\}, & A_{6}=\{\omega: p(s)<0, q(s) \geqslant p(s)\}, \\
A_{7}=\{\omega: p(s)<0,0<q(s)<-p(s)\}, & A_{8}=\{\omega: p(s)<0, q(s)>-p(s)\} .
\end{array}
$$

Положим

$$
\begin{aligned}
& z_{1}=-\frac{[p(s)+q(s)][p(s)-3 q(s)+\sqrt{D}]}{4 q(s)}, \\
& z_{2}=\frac{[p(s)+q(s)]^{2}}{2 q(s)}, \\
& z_{3}=-\frac{[p(s)+q(s)][p(s)-3 q(s)-\sqrt{D}]}{4 q(s)},
\end{aligned}
$$

где $D=[p(s)+q(s)][p(s)+9 q(s)]$.

Следуюшая теорема описывает изменение энергетического спектра оператора $\widetilde{H}_{1}$ в одномерном случае. 
Tеорема 1. А. Если $\omega \in A_{2} \cup A_{3}\left(\omega \in A_{4} \cup A_{8}\right)$, то оператор $\widetilde{H}_{1}$ имеет ровно два ЛПС $\varphi_{1}$ и $\varphi_{2}$ со значениями әнергии, соответственно равньми $z_{1}$ и $z_{2} \quad\left(z_{2}\right.$ u $\left.z_{3}\right)$, причем $z_{1}<z_{2}\left(z_{2}<z_{3}\right)$ u $z_{i}<m_{1}, \quad i=1,2 \quad\left(z_{j}>M_{1}, \quad j=2,3\right)$.

Б. Если $\omega \in A_{6}\left(\omega \in A_{5}\right)$, то оператор $\widetilde{H}_{1}$ имеет единственное ЛПС $\varphi$ со значением әнергии $z=z_{1} \quad\left(z=z_{3}\right)$, причем $z_{1}<m_{1} \quad\left(z_{3}>M_{1}\right)$.

В. Если $\omega \in A_{1} \cup A_{7}$, то оператор $\widetilde{H}_{1}$ не имеет ЛПС.

Приведем набросок доказательства теоремы 1. В одномерном случае уравнение $\Delta_{1}(z)=0$ эквивалентно совокупности уравнений

$$
1+q(s) \int_{T} \frac{(1-\cos t)^{2} d t}{p(s) h(t)-z}=0
$$

и

$$
1+q(s) \int_{T} \frac{\sin ^{2} t d t}{p(s) h(t)-z}=0 .
$$

Вычисляя значения интегралов в (8) и (9) в квадратурах для значений $z<m_{1} \quad(z>$ $\left.M_{1}\right)$, получаем два алгебраических уравнения. Исследуя полученные уравнения для значений $z<m_{1}\left(z>M_{1}\right)$ и учитывая условия сушествования решений, немедленно получаем доказательство теоремы 1.

В случае размерности $\nu=2$ введем следующие множества значений пар $\omega$ :

$$
\begin{aligned}
& Q_{1}=\{\omega: p(s)>0,-p(s) \leqslant q(s)<0\}, \quad Q_{2}=\{\omega: p(s)<0,0<q(s) \leqslant-p(s)\}, \\
& Q_{3}=\left\{\omega: p(s)>0,-\frac{100}{27} p(s) \leqslant q(s)<-p(s)\right\} \\
& Q_{4}=\left\{\omega: p(s)<0, \frac{25}{9} p(s) \leqslant q(s)<0\right\} \\
& Q_{5}=\left\{\omega: p(s)>0,0<q(s)<\frac{25}{9} p(s)\right\} \\
& Q_{6}=\left\{\omega: p(s)<0,-p(s) \leqslant q(s)<-\frac{25}{9} p(s)\right\} \\
& Q_{7}=\left\{\omega: p(s)>0,-\frac{100}{27} p(s) \leqslant q(s)<-p(s)\right\} \\
& Q_{8}=\left\{\omega: p(s)<0, \frac{100}{27} p(s) \leqslant q(s)<\frac{25}{9} p(s)\right\} \text {, } \\
& Q_{9}=\left\{\omega: p(s)>0, \frac{25}{9} p(s) \leqslant q(s)<\frac{100}{27} p(s)\right\} \text {, } \\
& Q_{10}=\left\{\omega: p(s)<0,-\frac{25}{9} p(s) \leqslant q(s)<-\frac{100}{27} p(s)\right\} \\
& Q_{11}=\left\{\omega: p(s)>0,-\frac{25}{9} p(s)<q(s)\right\}, \quad Q_{12}=\left\{\omega: p(s)<0, \frac{100}{27} p(s)<q(s)\right\}, \\
& Q_{13}=\left\{\omega: p(s)>0, \frac{100}{27} p(s) \leqslant q(s)\right\}, \quad Q_{14}=\left\{\omega: p(s)<0,-\frac{100}{27} p(s)<q(s)\right\} .
\end{aligned}
$$


Следуюшая теорема описьвает изменение энергетического спектра оператора $\widetilde{H}_{1}$ в двухмерном случае.

Tеорема 2. А. Если $\omega \in Q_{1} \cup Q_{2}$, то оператор $\widetilde{H}_{1}$ не имеет ЛПС.

Б. Если $\omega \in Q_{3} \cup Q_{4}\left(\omega \in Q_{5} \cup Q_{6}\right)$, то оператор $\widetilde{H}_{1}$ имеет единственное ЛПС $\varphi$ со значением энергии $z_{1}\left(z_{2}\right)$, причем $z_{1}<m_{2} \quad\left(z_{2}>M_{2}\right)$. Уровень этой әнергии невырожден.

B. Если $\omega \in Q_{7} \cup Q_{8}\left(\omega \in Q_{9} \cup Q_{10}\right)$, то оператор $\widetilde{H}_{1}$ имеет ровно два ЛПС $\varphi_{1}$ и $\varphi_{2}$ со значениями әнергии, соответственно равными $z_{1}$ и $z_{2}, z_{i}<m_{2}, \quad i=1,2$ $\left(\begin{array}{lll}z_{3} & \text { и } & z_{4},\end{array} z_{j}>M_{2}, j=3,4\right)$, и уровень этих энергий невырожден.

Г. Если $\omega \in Q_{11} \cup Q_{12} \quad\left(\omega \in Q_{13} \cup Q_{14}\right)$, то оператор $\widetilde{H}_{1}$ имеет три ЛПС $\varphi_{1}$, $\varphi_{2}$ и $\varphi_{3}$ со значениями әнергии, соответственно равныли $z_{1}, z_{2}, z_{3}\left(z_{4}, z_{5}, z_{6}\right)$, причем $z_{i}<m_{2}, \quad i=1,2,3 \quad\left(z_{j}>M_{2}, j=4,5,6\right)$. Энергетические уровни $z_{1}$ и $z_{3}$ $\left(\begin{array}{ll}z_{4} & и \\ z_{6}\end{array}\right)$ невырожденны, а уровень $z_{2}\left(z_{5}\right)$ двукратно вырожден.

ДОКАЗАТЕЛЬСТво. Функции

$$
\begin{aligned}
& \varphi(z)=\int_{T^{2}} \frac{\left(1-\cos t_{1}\right)\left(2-\cos t_{1}-\cos t_{2}\right)}{p(s) h(t)-z} d t \\
& \psi(z)=\int_{T^{2}} \frac{\sin ^{2} t_{1} d t}{p(s) h(t)-z}, \quad \theta(z)=\int_{T^{2}} \frac{\left(\cos t_{1}-\cos t_{2}\right)^{2} d t}{p(s) h(t)-z}
\end{aligned}
$$

являются возрастающими функциями $z$ при $z \notin\left[m_{2} ; M_{2}\right]$. Можно точно вычислить значения этих функций в точках $z=m_{2}$ и $z=M_{2}$. При $z<m_{2}$ и $p(s)>0$ функция $\varphi(z)$ возрастает от 0 до $1 / p(s)$, функция $\psi(z)$ возрастает от 0 до $9 /(25 p(s))$, а функшия $\theta(z)$ возрастает от 0 до $27 /(50 p(s))$. При $z>M_{2}$ и $p(s)>0$ эти функции также возрастают от $-\infty$ до 0 , от $-9 /(25 p(s))$ до 0 и от $-27 /(50 p(s))$ до 0 , соответственно. Если $p(s)<0$ и $z<m_{2}$, то эти функции возрастают от 0 до $\infty$, от 0 до $-9 /(25 p(s))$ и от 0 до $-27 /(50 p(s))$, соответственно. При $p(s)<0$ и $z>M_{2}$ функции $\varphi(z), \psi(z)$ и $\theta(z)$ возрастают от $1 / p(s)$ до 0 , от $9 /(25 p(s))$ до 0 и от $27 /(50 p(s))$ до 0 , соответственно. Исследуя уравнение $\Delta_{2}(z)=0$ вне области непрерывного спектра, немедленно получаем утверждение теоремы.

В случае $\nu=3$ введем следуюшие обозначения:

$$
\begin{aligned}
a & =\int_{T^{3}} \frac{\sin ^{2} t_{1} d t_{1} d t_{2} d t_{3}}{3-\cos t_{1}-\cos t_{2}-\cos t_{3}}=\int_{T^{3}} \frac{\sin ^{2} t_{1} d t_{1} d t_{2} d t_{3}}{3+\cos t_{1}+\cos t_{2}+\cos t_{3}}, \\
b & =\int_{T^{3}} \frac{\left(\cos t_{1}-\cos t_{2}\right)^{2} d t_{1} d t_{2} d t_{3}}{3-\cos t_{1}-\cos t_{2}-\cos t_{3}}=\int_{T^{3}} \frac{\left(\cos t_{1}-\cos t_{2}\right)^{2} d t_{1} d t_{2} d t_{3}}{3+\cos t_{1}+\cos t_{2}+\cos t_{3}}
\end{aligned}
$$

Видно, что $0<a<b<1$ и $2 a<b$. Теперь для случая $\nu=3$ введем следующие подмножества $\Omega$ : 


$$
\begin{aligned}
& Q_{1}=\{\omega: p(s)>0,-p(s)<q(s)<0\}, \quad Q_{2}=\left\{\omega: p(s)>0,0<q(s)<\frac{p(s)}{3}\right\} \\
& Q_{3}=\left\{\omega: p(s)<0, \frac{p(s)}{3}<q(s)<0\right\}, \quad Q_{4}=\{\omega: p(s)<0,0<q(s)<-p(s)\}, \\
& Q_{5}=\left\{\omega: p(s)>0,-\frac{2 p(s)}{b}<q(s) \leqslant-p(s)\right\}, \\
& Q_{6}=\left\{\omega: p(s)<0, \frac{2 p(s)}{b}<q(s) \leqslant \frac{p(s)}{3}\right\}, \\
& Q_{7}=\left\{\omega: p(s)>0, \frac{p(s)}{3}<q(s) \leqslant \frac{2 p(s)}{b}\right\}, \\
& Q_{8}=\left\{\omega: p(s)<0,-p(s)<q(s) \leqslant-\frac{2 p(s)}{b}\right\} \\
& Q_{9}=\left\{\omega: p(s)>0,-\frac{p(s)}{a} \leqslant q(s)<-\frac{2 p(s)}{b}\right\}, \\
& Q_{10}=\left\{\omega: p(s)<0, \frac{p(s)}{a}<q(s) \leqslant \frac{2 p(s)}{b}\right\}, \\
& Q_{11}=\left\{\omega: p(s)>0, \frac{2 p(s)}{b} \leqslant q(s)<\frac{p(s)}{a}\right\}, \\
& Q_{12}=\left\{\omega: p(s)<0,-\frac{2 p(s)}{b} \leqslant q(s)<-\frac{p(s)}{a}\right\}, \\
& Q_{13}=\left\{\omega: p(s)>0,-\frac{p(s)}{a} \leqslant q(s)\right\}, \quad Q_{14}=\left\{\omega: p(s)<0, \frac{p(s)}{a} \leqslant q(s)\right\} \\
& Q_{15}=\left\{\omega: p(s)>0, \frac{p(s)}{a} \leqslant q(s)\right\}, \quad Q_{16}=\left\{\omega: p(s)<0,-\frac{p(s)}{a} \leqslant q(s)\right\} .
\end{aligned}
$$

Teоpema 3. А. Пусть $\omega \in Q_{1} \cup Q_{2} \cup Q_{3} \cup Q_{4}$. Тогда оператор $\widetilde{H}_{1}$ не имеет ЛПС.

Б. Пусть $\omega \in Q_{5} \cup Q_{6} \quad\left(\omega \in Q_{7} \cup Q_{8}\right)$. Тогда оператор $\widetilde{H}_{1}$ имеет единственное ЛПС $\varphi$ со значением энергии $z<m_{3} \quad\left(z>M_{3}\right)$. Уровень этой әнергии невырожден.

В. Пусть $\omega \in Q_{9} \cup Q_{10}\left(\omega \in Q_{11} \cup Q_{12}\right)$. Тогда оператор $\widetilde{H}_{1}$ имеет два ЛПС $\varphi_{1}$ и $\varphi_{2}$ со значениями әнергии, соответственно равными $z_{1}$ и $z_{2}\left(\begin{array}{lll}z_{3} & \text { и } \\ z_{4}\end{array}\right)$, причем $z_{i}<m_{3}, \quad i=1,2\left(z_{j}>M_{3}, j=3,4\right)$. Кроме того, әнергетический уровень $z_{1}\left(z_{3}\right)$ невырожден, а уровень $z_{2}\left(z_{4}\right)$ двукратно вырожден.

Г. Пусть $\omega \in Q_{13} \cup Q_{14} \quad\left(\omega \in Q_{15} \cup Q_{16}\right)$. Тогда оператор $\widetilde{H}_{1}$ имеет ровно три ЛПС $\varphi_{1}, \varphi_{2}$ и $\varphi_{3}$ со значениями әнергии, соответственно равньми $z_{1}, z_{2}$, $z_{3}\left(z_{4}, z_{5}, z_{6}\right)$. Причем $z_{i}<m_{3}, i=1,2,3 \quad\left(z_{j}>M_{3}, j=4,5,6\right)$. Кроме того, уровень $z_{1}\left(z_{4}\right)$ невырожден, уровень $z_{2}\left(z_{5}\right)$ двукратно вырожден, а уровень $z_{3}$ $\left(z_{6}\right)$ трехкратно вырохсден. 
Теорема 3 доказывается на основании монотонности функций

$$
\begin{gathered}
\varphi(z)=\int_{T^{3}} \frac{\left(1-\cos t_{1}\right)\left(3-\cos t_{1}-\cos t_{2}-\cos t_{3}\right) d t}{p(s) h(t)-z} \\
\psi(z)=\int_{T^{3}} \frac{\sin ^{2} t_{1} d t}{p(s) h(t)-z}, \quad \theta(z)=\int_{T^{3}} \frac{\left(\cos t_{1}-\cos t_{2}\right)^{2} d t}{p(s) h(t)-z}
\end{gathered}
$$

при значениях $z \notin\left[m_{3} ; M_{3}\right]$. Далее воспользуемся значением интегралов Ватсона [5]. При этом необходимо учесть, что в рассматриваемом случае мера нормирована.

Аналогично можно доказать, что в $\nu$-мерной решетке система имеет не более трех типов ЛПС (без учета кратности вырождений их энергетических уровней) со значениями энергии $z_{i} \notin\left[m_{\nu} ; M_{\nu}\right]$. Кроме того, при $i=1,2,3$ эти энергетические уровни соответственно невырожденны, $\nu,(\nu-1)$-кратно вырожденны. Можно найти и область сушествования этих ЛПС.

Теперь рассмотрим случай $p(s) \equiv 0$.

Если $p(s) \equiv 0$ и $J_{n} \neq 0, n=1,2, \ldots, 2 s$, то функция $\Delta_{\nu}(z)$ принимает вид

$$
\Delta_{\nu}(z)=\operatorname{det} A \cdot \operatorname{det} B
$$

где

$$
A=\left(\begin{array}{cccccc}
a_{1} & b_{1} & b_{1} & b_{1} & \ldots & b_{1} \\
a_{2} & b_{2} & 0 & 0 & \ldots & 0 \\
a_{2} & 0 & b_{2} & 0 & \ldots & 0 \\
a_{2} & 0 & 0 & b_{2} & \ldots & 0 \\
\ldots & \ldots & \ldots & \ldots & \ldots & \ldots \\
\ldots & \ldots & \ldots & \ldots & \ldots & \ldots \\
a_{2} & 0 & 0 & 0 & \ldots & \ldots \\
b_{2}
\end{array}\right), \quad B=\left(\begin{array}{ccccc}
b_{2} & 0 & 0 & \ldots & 0 \\
0 & b_{2} & 0 & \ldots & 0 \\
0 & 0 & b_{2} & \ldots & 0 \\
\ldots & \ldots & \ldots & \ldots & \ldots \\
\ldots & \ldots & \ldots & \ldots & \ldots \\
0 & 0 & 0 & \ldots & b_{2}
\end{array}\right)
$$

- матрица порядка $(\nu+1) \times(\nu+1)$, диагональная матрица порядка $\nu \times \nu$, соответственно. Здесь $a_{1}=1-\nu q(s) / z, a_{2}=q(s) /(2 z), b_{1}=q(s) / z, b_{2}=1-q(s) /(2 z)$.

Имеет место

Tеорема 4. Пусть $p(s) \equiv 0 u J_{n} \neq 0, n=1,2, \ldots, 2 s$. Тогда оператор $\widetilde{H}_{1}$ имеет ровно два ЛПС (без учета кратности вырожсдений их энергии) $\varphi_{1}$ и $\varphi_{2}$ со значениями энергии, соответственно равными $z_{1}=q(s) / 2, z_{2}=(2 \nu+1) q(s) / 2$. При этом энергетический уровень $z_{1}(2 \nu-1)$-кратно вырожден, а уровень $z_{2}$ невырожден.

ДокАЗАТЕЛЬСтво. Уравнение $\Delta_{\nu}(z)=0$ эквивалентно совокупности уравнений

$$
b_{2}^{2 \nu-1}=0
$$

$$
a_{1} b_{2}-\nu a_{2} b_{1}=0 .
$$


Уравнение (10) имеет корень $z=q(s) / 2$, и ясно, что его кратность равна $2 \nu-1$, а уравнение (11) имеет решение $z=z_{2}$. Следовательно, для произвольных значений $\nu$ система обладает не более чем тремя типами ЛПС.

Полученные результаты показьвают, что в случае негейзенберговской изотропной примесной ферромагнитной модели при $p(s) \equiv 0$ спектр и ЛПС одномагнонных систем качественно отличаются от соответствуюших характеристик изотропной примесной ферромагнитной модели Гейзенберга. Вообще говоря, в случае негейзенберговской изотропной примесной ферромагнитиной модели одномагнонных систем имеется не более чем $2 \nu$ ЛПС (с учетом кратности вырождений их энергии).

Благодарности. Автор выражает свою благодарность профессору В. И. Чилину за полезные обсуждения.

\section{Список литературы}

[1] Ю. А. Изюмов, М. В. Медведев. ЖЭТФ. 1965. Т. 48. № 2. С. 574; В. В. Ганн, Л. Г. Зазунов. ФТT. 1973. Т. 15. № 2. С. 3535; Y.-L. Wang, H. Callen. Phys. Rev. 1967. V. 160. № 2. P. 358; T. Oguchi, I. Ono. J. Phys. Soc. Japan. 1969. V. 26. № 1. P. 32; T. Wolfram, J. Callaway. Phys. Rev. 1963. V. 130. № 6. P. 2207; I. Ono, Y. Endo. Phys. Rev. Lett. A. 1972. V. 41. № 5. P. 440.

[2] С. М Ташпулатов. ТМФ. 2001. Т. 126. № 3. С. 482.

[3] E. Schrödinger. Proc. Roy. Irish. Acad. A. 1941. V. 48. P. 39.

[4] М. Рид, Б. Саймон. Методы современной математической физики. Т. 1. Функциональный анализ. М.: Мир, 1977.

[5] В. В. Вальков, С. Г. Овчинников, О. П. Петраковский. ФТТ. 1988. Т. 30. № 10. С. 3044.

Поступила в редакцию 19.VI.2003 г., после доработки 7.VI.2004 г. 